\title{
Bestimmung der hydraulischen Durchlässigkeiten eines Sandsteins mithilfe eines Luftpermeameters
}

\author{
Sina Hale ${ }^{1}$ (D) Philipp Blum ${ }^{1}$ \\ Eingegangen: 6. Juli 2021 / Überarbeitet: 17. November 2021 / Angenommen: 6. Dezember 2021 / Online publiziert: 10. Januar 2022 \\ (c) Der/die Autor(en) 2022
}

\section{Zusammenfassung}

Ziel dieser Studie ist die Bestimmung der hydraulischen Durchlässigkeiten eines Sandsteins unter Berücksichtigung der Gesteinsmatrix sowie einer Einzelkluft unter Verwendung eines tragbaren Luftpermeameters. Hierfür wurde der fluviatil-äolisch abgelagerte Bebertaler Sandstein des Oberen Rotliegenden in Sachsen-Anhalt untersucht. Es wurden die Matrixpermeabilitäten der unterschiedlichen Faziesbereiche sowie die Kluftöffnungsweiten entlang einer Schichtfuge bestimmt. Die ermittelten hydraulischen Durchlässigkeiten der Sandsteinmatrix liegen dabei zwischen $1,0 \cdot 10^{-7}$ und $9,2 \cdot 10^{-10} \mathrm{~m} / \mathrm{s}$, allerdings weisen nur 3 von insgesamt 298 Messpunkten einen $k_{f}$-Wert von $>7,4 \cdot 10^{-8} \mathrm{~m} / \mathrm{s}$ bzw. eine Permeabilität von $>10$ $\mathrm{mD}$ auf. Diese gehören zur homogenen und höher durchlässigen äolischen Fazies. Die bestimmte mittlere Öffnungsweite der Schichtfuge liegt bei $82 \pm 12 \mu \mathrm{m}$. Mithilfe der ermittelten hydraulischen Eigenschaften konnte somit die effektive hydraulische Durchlässigkeit des untersuchten Sandsteins bestimmt werden. Unsere Ergebnisse verdeutlichen die praktische und robuste Anwendbarkeit des verwendeten Luftpermeameters zur Bestimmung der hydraulischen Durchlässigkeiten von Sandsteinen sowohl im Labor als auch im Gelände.

Schlüsselwörter Matrixpermeabilität · Kluftöffnungsweite $\cdot$ Sandstein $\cdot$ Effektive hydraulische Durchlässigkeit

\section{Determination of the hydraulic conductivities of a sandstone using an air permeameter}

\begin{abstract}
The objective of the current study is to determine hydraulic conductivities of a sandstone matrix and a single fracture using a portable air permeameter. For this purpose, the fluvial-aeolian Bebertal sandstone from the Upper Rotliegend in Saxony-Anhalt is investigated. Matrix permeabilities of the different facies and hydraulic apertures along a bedding joint are determined. The estimated hydraulic conductivities of the sandstone matrix range between $1.0 \cdot 10^{-7}$ and $9.2 \cdot 10^{-10} \mathrm{~m} / \mathrm{s}$, but only 3 out of 298 measurement points show $K$ values of $>7.4 \cdot 10^{-8} \mathrm{~m} / \mathrm{s}$ or permeabilities of $>10 \mathrm{mD}$. These belong to the more homogeneous and higher permeable aeolian facies. The estimated mean hydraulic fracture aperture is $82 \pm 12 \mu \mathrm{m}$. Using both hydraulic parameters, the effective hydraulic conductivity of the studied sandstone can be determined. Our results illustrate the practical and robust applicability of the air permeameter for determining hydraulic conductivities of sandstones in the laboratory and in the field.
\end{abstract}

Keywords Matrix permeability $\cdot$ Fracture aperture $\cdot$ Sandstone $\cdot$ Effective hydraulic conductivity

\section{Einführung}

Sina Hale

sina.hale@kit.edu

1 Institut für Angewandte Geowissenschaften (AGW), Karlsruher Institut für Technologie (KIT), Adenauerring 20b, 76131 Karlsruhe, Deutschland
Sandsteine und deren hydraulische Eigenschaften spielen eine wichtige Rolle bei der Energiewende in Deutschland. Sie dienen als Reservoir- und Speichergesteine für Kohlenwasserstoffe, Gas- und Wasserstoffspeicher, aber auch für thermische Reservoire und Speicher in Form sogenannter Aquiferspeicher (z.B. Kühn et al. 2012; Huq et al. 2015; 
Kabuth et al. 2017; Fleuchaus et al. 2021). Darüber hinaus sind Sandsteine auch für die regionale Trinkwasserversorgung von großer Bedeutung (z. B. Hanauer und Söll 1996; Baier et al. 2016; Meurer und Banning 2019), und zahlreiche größere Grundwasserkontaminationen in Deutschland befinden sich ebenfalls in unterschiedlichen Sandsteinformationen (z.B. Marczinek et al. 2006; Brinkmann et al. 2014; Altenbockum et al. 2018). Die Bestimmung der hydraulischen Durchlässigkeiten dieser Grundwasserleiter bildet dabei immer eine zentrale Aufgabe. Typischerweise werden diese hydraulischen Eigenschaften im Labor oder auch im Feld mithilfe von aufwendigen Pump- und Tracerversuchen bestimmt (z. B. Novakowski und Lapcevic 1994; Weede und Hötzl 2005; Zeeb et al. 2010; Altenbockum et al. 2018).

Ergänzend zu diesen integrierenden, zeitlich und technisch aufwendigen hydraulischen Tests können die lokalen hydraulischen Durchlässigkeiten auf der kleinmaßstäblichen Skala (cm-Skala) auch mithilfe eines tragbaren Luftpermeameters bestimmt werden. Diese Messgeräte werden schon seit mehreren Jahrzehnten im Labor und im Feld zur Bestimmung der hydraulischen Durchlässigkeit eingesetzt. Huysmans et al. (2008) geben einen kompakten und historischen Überblick über deren Anwendung und Entwicklung. Durch die Einführung kommerzieller Luftpermeameter hat die Anwendung in den letzten Jahren stark zugenommen. Die Anwendung findet sowohl in Locker- als auch in Festgesteinen statt. Rogiers et al. (2014a, b) konnten mithilfe des Luftpermeameters räumlich hochauflösende Aquiferheterogenitäten der hydraulischen Durchlässigkeiten an unkonsolidierten Sanden bestimmen. Des Weiteren verglichen Rogiers et al. (2013) $k_{f}$-Werte aus Aufschlüssen und Bohrlöchern. Hier zeigte sich, dass die $k_{f}$-Werte aus den Aufschlüssen, die mit dem Luftpermeameter bestimmt wurden, aufgrund ihrer höheren lokalen Auflösung tendenziell höher waren als die $k_{f}$-Werte, die mittels hydraulischer Pumpversuche in Bohrlöchern bestimmt wurden. Auch andere Studien beschreiben eine Skalenabhängigkeit der hydraulischen Durchlässigkeit, teils über mehrere Größenordnungen von Kernproben bis hin zur Feldskala (z. B. Mallants et al. 1997; Bagarello und Provenzano 1996; Schulze-Makuch et al. 1999; Whitaker und Smart 2000).

Filomena et al. (2014) untersuchten die Bestimmung der hydraulischen Durchlässigkeit mit verschiedenen kommerziellen Permeametern, u. a. auch dem tragbaren TinyPerm II, der auch in den vorher genannten Arbeiten von Rogiers et al. (2013, 2014a, b) zum Einsatz kam, sowie mit zwei Luftpermeametern nach dem Prinzip der Hassler Zelle an verschiedenen Sandsteinen. Für die Messung mit der Hassler Zelle muss die Gesteinsprobe jedoch exakt in Abhängigkeit der Maße des Probenhalters präpariert werden, um Umläufigkeiten während des Versuchs zu vermeiden. Die Ergebnisse dieser Untersuchungen zeigen, dass Abwei- chungen zwischen den unterschiedlichen Apparaturen und Methoden existieren, diese jedoch auch korrigiert werden können, um so eine sehr gute Vergleichbarkeit zu erreichen. Darüber hinaus wurde der tragbare Luftpermeameter (TinyPerm II) an Vulkanböden des Solfatara Kraters in Italien (Montanaro et al. 2017), an Festgesteinen wie Andesit (Farquharson et al. 2015), Kalksteinen (De Boever et al. 2016) und Tonsteinen (Hale et al. 2021), oder auch an Störungszonen erfolgreich zur Bestimmung der Permeabilität eingesetzt (Petrie et al. 2014).

Der Luftpermeameter TinyPerm 3 von New England Research ermöglicht jedoch auch die Bestimmung der hydraulischen Kluftöffnungsweite $a_{\mathrm{h}}$. In Hale et al. (2020) wurde ein Vergleich von drei unterschiedlichen Methoden (Luftpermeameter, Mikroskopkamera und Laserscanner) zur Ermittlung von $a_{\mathrm{h}}$ an einer Sandsteinschichtfuge durchgeführt. Hier konnte gezeigt werden, dass der Luftpermeameter die robustesten Ergebnisse zur Bestimmung von $a_{\mathrm{h}}$ liefert. Mithilfe der beiden anderen Methoden (Mikroskopkamera und Laserscanner) konnte diese lediglich indirekt über die Bestimmung der mechanischen Kluftöffnungsweite $a_{\mathrm{m}}$ und die Kluftrauigkeit hergeleitet werden. In einer Studie von Cheng et al. (2020) konnte die Bestimmung der hydraulischen Kluftöffnungsweite mithilfe des Luftpermeameters validiert werden. In dieser Studie wurde dazu ein Vergleich von drei Methoden (Durchflusszelle, Luftpermeameter und Mikroskopkamera) zur Bestimmung von $a_{\mathrm{h}}$ an geklüfteten Sandsteinkernproben (Durchmesser $30 \mathrm{~mm}$ und Länge $40 \mathrm{~mm}$ ) durchgeführt. Der Vergleich der bestimmten hydraulischen Kluftöffnungsweiten, die zwischen 8 und $66 \mu \mathrm{m}$ lagen, zeigte eine exzellente Übereinstimmung $\left(\mathrm{R}^{2}=0,998\right)$. Somit konnte mithilfe der Arbeiten von Filomena et al. (2014) und Cheng et al. (2020) die verlässliche Bestimmung der Matrixpermeabilität und der hydraulischen Kluftöffnungsweite mithilfe des Luftpermeameters TinyPerm 3 eindeutig nachgewiesen und mit anderen Standardmethoden validiert werden. Nach unserem Kenntnisstand wurde der Luftpermeameter jedoch noch nicht zur Bestimmung der effektiven hydraulischen Durchlässigkeit, d.h. für eine kombinierte Betrachtung von Matrix und Kluft, eingesetzt.

Ziel dieser Arbeit ist es daher, den Luftpermeameter zur Bestimmung der effektiven Durchlässigkeit an einem Sandstein anzuwenden. Für diese Untersuchungen verwenden wir einen Probeblock des Bebertaler Sandsteins, der eine Schichtfuge beinhaltet. Mithilfe dieses Sandsteinblocks ist es daher möglich, die Matrixpermeabilitäten der unterschiedlichen Fazies sowie die hydraulische Kluftöffnungsweite entlang der Schichtfuge zu bestimmen, um somit die mittlere effektive Durchlässigkeit der Sandsteinprobe zu berechnen. 


\section{Material und Methoden}

\section{Sandsteinprobe}

Der in dieser Studie untersuchte Sandsteinblock stammt aus dem Steinbruch Bebertal, der $28 \mathrm{~km}$ NW von Magdeburg liegt (Geologische Karte von Erxleben, Blatt 3733, Koordinaten: R 4452 700/H 5789 800, Abb. 1). Der Aufschluss in Bebertal ermöglicht den Einblick in die fluviatil-äolische Fazies des Flechtinger Bausandsteins und gehört der Parchim-Formation (Havel-Subgruppe) des südlichen Permbeckens an (Fischer et al. 2007, 2012). Heidsiek et al. (2020) untersuchten denselben Sandsteinblock hinsichtlich der unterschiedlichen diagenetischen Fazies. In dieser Untersuchung wurde die räumliche Verteilung der Porosität, der Matrixpermeabilität, die mit dem Luftpermeameter TinyPerm 3 bestimmt wurde, sowie die räumliche Verteilung der dazugehörigen Zementminerale wie Quarz und Kalzit auf der cm-Skala analysiert. Mithilfe des Softwareprogramms Petrel 2017 von Schlumberger konnten diese Eigenschaften in einem 3D-Modell interpoliert und visualisiert werden. Die Ergebnisse illustrieren die Abhängigkeit der Porositäten und Permeabilitäten von der Kalzitzementmenge (Heidsiek et al. 2020).

\section{Luftpermeameter}

In dieser Studie wurde der Luftpermeameter TinyPerm 3 verwendet (New England Research, Inc. 2015, Abb. 2a). Hierbei handelt es sich um ein tragbares Messgerät mit einem Gewicht von $1,2 \mathrm{~kg}$, mit dem die Matrixpermeabilität sowie die hydraulische Kluftöffnungsweite zwischen $10^{-15}$ und $10^{-11} \mathrm{~m}^{2}$ (1 mD und 10Darcy) bzw. 10 und $2000 \mu \mathrm{m}$ bestimmt werden kann. Die Messunsicherheit beträgt $\pm 5 \%$ für die Matrixpermeabilität und $\pm 1,4 \%$ für die Kluftöffnungsweite (Brown und Smith 2013). In Abb. 2b ist die Messung beispielhaft für die Öffnungsweitenbestimmung dargestellt, das Messprinzip sowie die Auswertungsmethodik ist jedoch in beiden Fällen identisch. Nach Anpressen der abdichtenden Gummispitze an die Probe wird zum Start der Messung ein Vakuum im Innern des Messgeräts erzeugt. Dieser Druckimpuls führt dazu, dass Luft von der Probe ins Innere des Geräts strömt und einen Druckausgleich bewirkt, dessen zeitliche Entwicklung durch die vorliegende hydraulische Öffnungsweite bzw. die Matrixpermeabilität gesteuert wird. Das Messprinzip ist in Hale et al. (2020) und Cheng et al. (2020) ausführlich beschrieben. Die Auswertung der Messung erfolgt automatisch und gerätintern. Während der Messung wird der initial aufgebrachte Druck $P_{0}(t)$ und der resultierende Durchfluss $Q(t)$ zeitabhängig aufgezeichnet und mithilfe der folgenden Antwortfunktion $H$ automatisch durch die Mikrocontroller-Einheit des Luftpermeameters ausgewertet (Brown und Smith 2013):

$H=\frac{\int_{-\infty}^{\infty} Q(t) d t}{\int_{-\infty}^{\infty} P_{0}(t) d t^{\prime}}$

Die Antwortfunktion hängt dabei im Wesentlichen von der Permeabilität der Matrix bzw. der Öffnungsweite der Kluft, aber auch von der Probengeometrie ab. Diese Parameter sind aber z.T. schwer zu ermitteln, daher wurden zur Bestimmung der hydraulischen Eigenschaften empirische Kalibrierungen durchgeführt. Diese sind ausführlich von Brown und Smith (2013) beschrieben. Die empirische
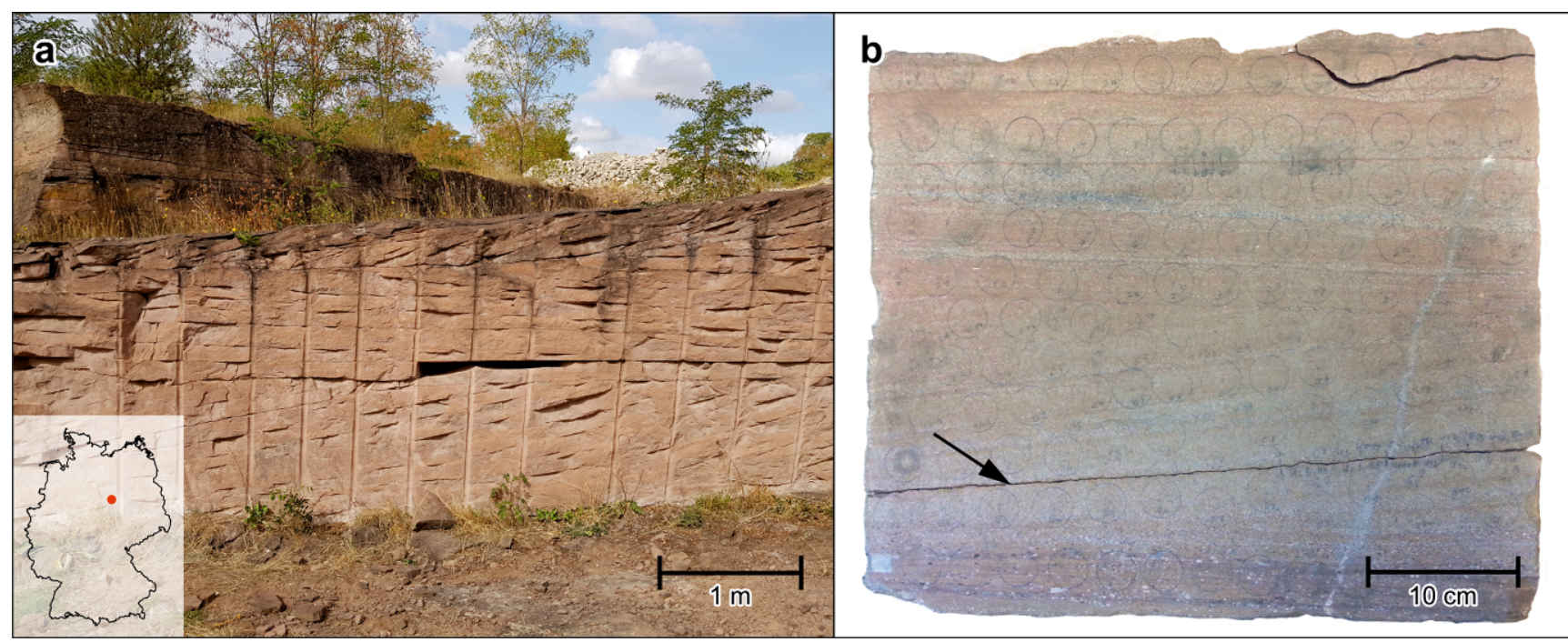

Abb. 1 a Aufschluss des Flechtinger Sandsteins im Steinbruch Bebertal und b untersuchter Sandsteinblock $(\mathrm{Maße} 40 \mathrm{~cm} \times 45 \mathrm{~cm} \times 11,5 \mathrm{~cm}) \mathrm{mit}$ Schichtfuge (schwarzer Pfeil)

Fig. 1 a Outcrop of the Flechtinger sandstone in the Bebertal quarry and b the examined sandstone block $($ dimensions $40 \mathrm{~cm} \times 45 \mathrm{~cm} \times 11.5 \mathrm{~cm})$ with bedding joint (black arrow) 


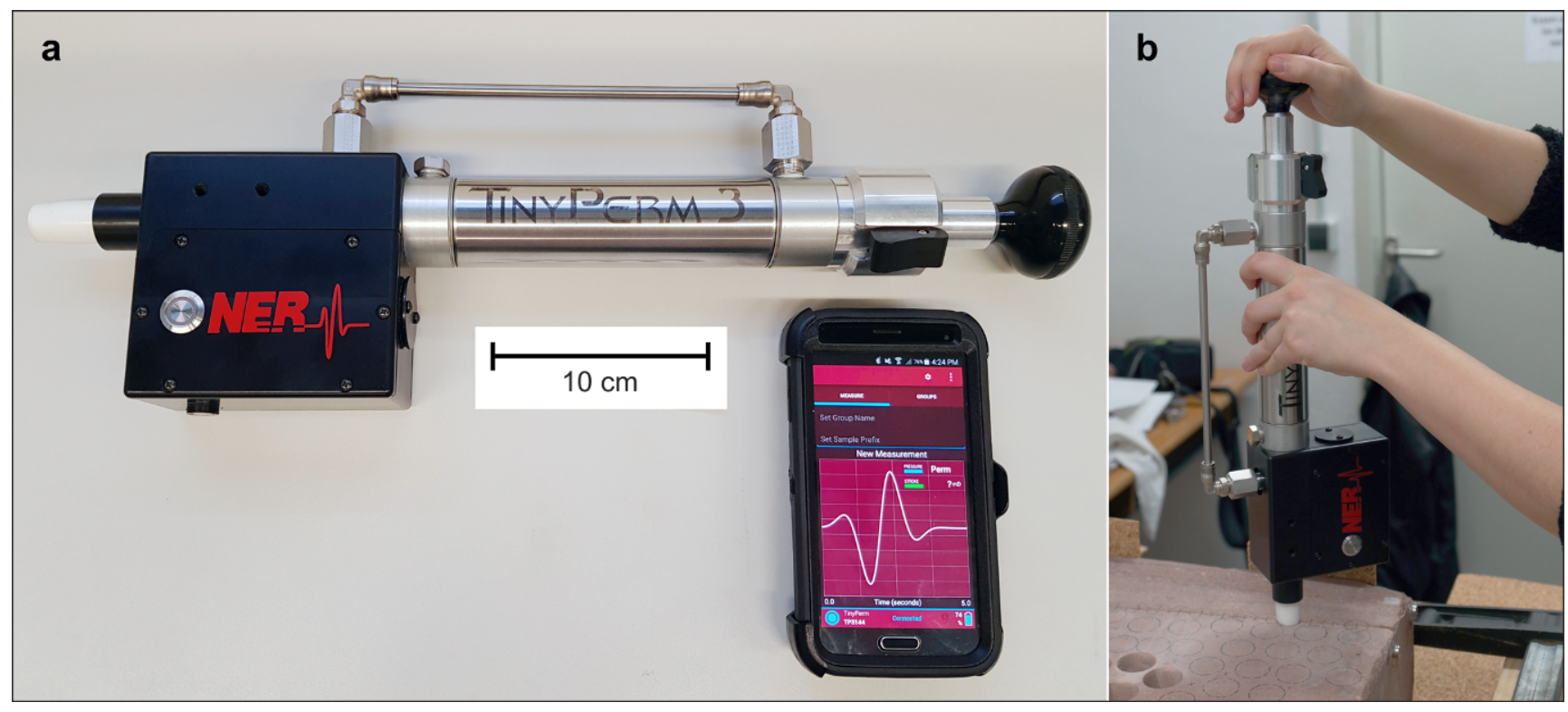

Abb. 2 a Luftpermeameter TinyPerm 3 mit Smartphone zur Steuerung und Auswertung, b Messung der hydraulischen Kluftöffnungsweite mithilfe des Luftpermeameters

Fig. 2 a Air permeameter TinyPerm 3 with smartphone for control and evaluation, b measurement of the hydraulic fracture aperture with the air permeameter

Kalibrierung der hydraulischen Kluftöffnungsweite erfolgte hierbei mithilfe einer künstlichen Kluft bestehend aus zwei polierten und planparallelen Granitproben. Die daraus ermittelte empirische Formel lautet wie folgt (Brown und Smith 2013):

$T=-1,5 \log _{10}\left(a_{\mathrm{TP}}\right)+8,29$

mit $a_{\mathrm{TP}}$ der hydraulischen Kluftöffnungsweite bestimmt mit dem TinyPerm, welche eine planparallele Kluft annimmt, und dem Wert $T$, der durch die Messung mit dem TinyPerm ermittelt wird und dem dekadischen Logarithmus der finalen Antwortfunktion entspricht, wenn der Druck im Luftpermeameter wieder den anfänglichen Umgebungsdruck erreicht hat. Da die Kalibrierung der hydraulischen Öffnungsweite an Granitproben mit sehr geringer Matrixpermeabilität durchgeführt wurde, kann davon ausgegangen werden, dass die Antwortfunktion in diesem Fall nahezu ausschließlich den Druckimpuls der Kluft wiedergibt. Bei der Messung von Kluftöffnungsweiten in Gesteinen mit hoher Matrixpermeabilität ist diese Voraussetzung jedoch nur bedingt gegeben. Hier wäre eine zusätzliche Kalibrierung des Luftpermeameters mit durchlässigerem geklüfteten Probematerial zu empfehlen, um den Effekt der Matrixpermeabilität auf den Druckimpuls zu berücksichtigen.

Für die Herleitung der empirischen Kalibrierkurve für die Bestimmung der Matrixpermeabilität wurden verschiedene Sedimentgesteine verwendet (Brown und Smith 2013):
$T=-0,82 \log _{10}\left(K_{\mathrm{m}}\right)+12,87$

Für jeden Messpunkt entlang der Kluft im Sandsteinblock wurden zehn Wiederholungsmessungen durchgeführt, für die Messung der Matrixpermeabilität variierte die Anzahl der Wiederholungsmessungen pro Messpunkt zwischen drei und zehn, jeweils abhängig von der Reproduzierbarkeit der Werte.

\section{Effektive hydraulische Durchlässigkeit}

Mithilfe des Luftpermeameters kann die Matrixpermeabilität $K_{\mathrm{m}}$ in Darcy sowie die hydraulische Kluftöffnungsweite $a_{\mathrm{TP}}$ in $\mu \mathrm{m}$ direkt bestimmt werden. Um die effektive hydraulische Durchlässigkeit des gesamten untersuchten Sandsteinblocks $k_{\text {eff }}$ zu berechnen, müssen beide hydraulische Größen des TinyPerm 3 in hydraulische Durchlässigkeiten in $\mathrm{m} / \mathrm{s}$ wie folgt umgerechnet werden:

Hydraulische Durchlässigkeit der Matrix $k_{\mathrm{m}}$ :

$k_{\mathrm{m}}=\frac{K_{\mathrm{m}} \rho g}{\eta}$

Hydraulische Durchlässigkeit der Kluft im Sandsteinblock $k_{\text {fr }}$ nach Cao et al. (2016):

$k_{\mathrm{fr}}=\frac{g a_{\mathrm{TP}}^{3}}{12 v L}$

mit $\rho$ der Dichte von Wasser bei einer Grundwassertemperatur von $10^{\circ} \mathrm{C}\left(=999,7 \mathrm{~kg} / \mathrm{m}^{3}\right), g$ der Gravitationsbeschleunigung mit $9,81 \mathrm{~m} / \mathrm{s}^{2}, \eta$ der dynamischen Viskosität von 
Abb. 3 a Ergebnisse der Bestimmung der räumlich verteilten Matrixpermeabilitäten (fluviatile und äolische Fazies, Darstellung der 122 Messpunkte auf der Vorderseite des Sandsteinblocks) und $\mathbf{b}$ der hydraulischen Kluftöffnungsweiten entlang der Schichtfuge (Abschnitte A1 bis A21) mit Angaben der jeweils ermittelten Mittelwerte und der dazugehörigen Standardabweichungen

Fig. 3 a Results of the determination of the spatially distributed matrix permeabilities (fluvial and aeolian facies, representation of the 122 measuring points on the front side of the sandstone block) and $\mathbf{b}$ the hydraulic fracture apertures along the bedding joint (segments A1 to A21) with mean values and standard deviations

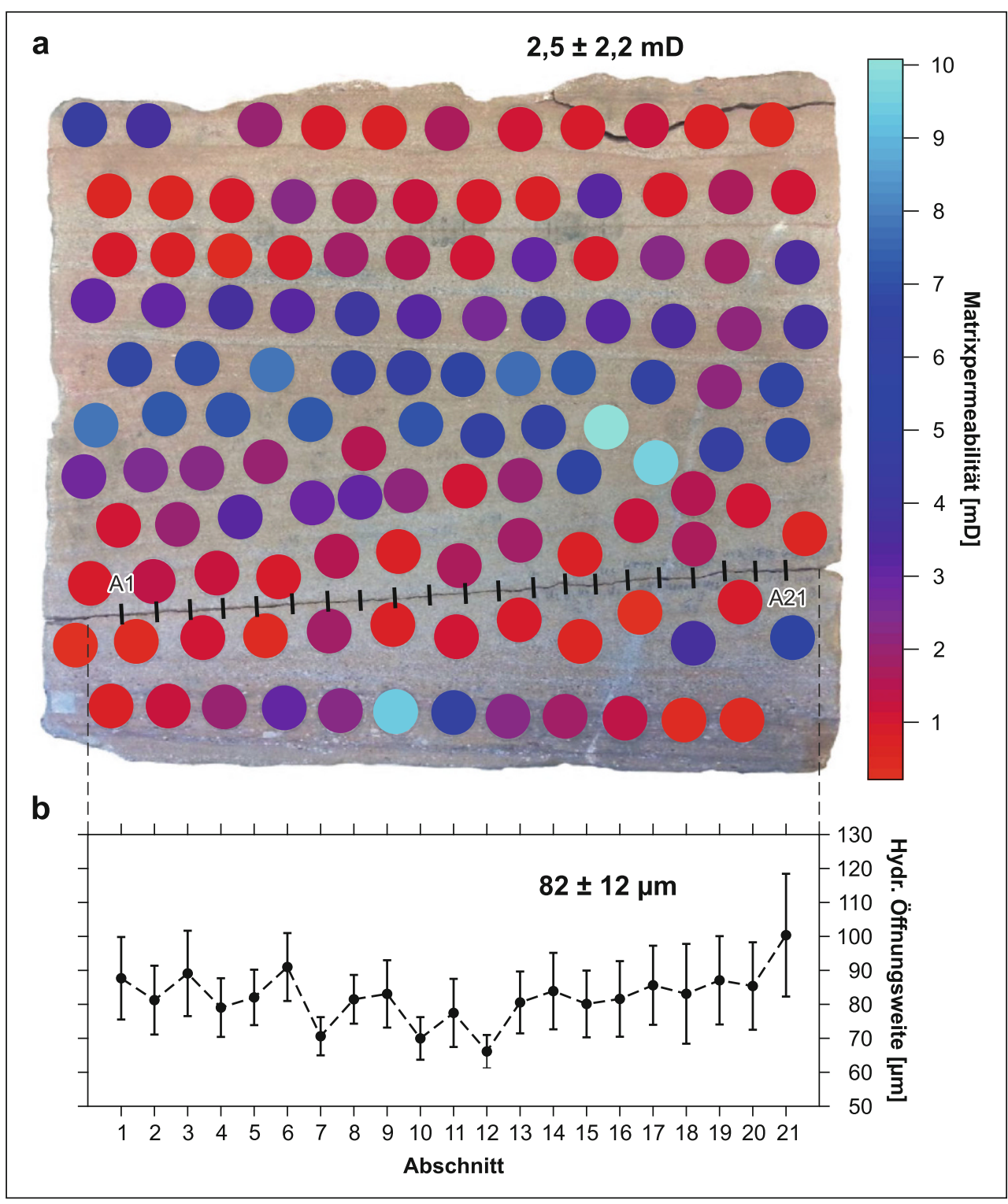

Wasser bei $10^{\circ} \mathrm{C}\left(=1,3064 \cdot 10^{-3} \mathrm{~kg} / \mathrm{m} \mathrm{s}\right.$ nach Sato 2005$)$ und $v$ der kinematischen Viskosität von Wasser bei $10^{\circ} \mathrm{C}$ $\left(=1,307 \cdot 10^{-6} \mathrm{~m}^{2} / \mathrm{s}\right.$ mit $\left.v=\eta / g\right)$. Die Distanz $L$ beschreibt die Distanz zu den beiden Randbedingungen, hier die Distanz zwischen dem oberen und unteren Rand des Sandsteinblocks $(0,4 \mathrm{~m})$. Bei Betrachtung einer Kluftschar entspricht $L$ dem mittleren Kluftabstand (z. B. Bai und Elsworth 1994). Ein Darcy wird mit $9,86923 \cdot 10^{-13} \mathrm{~m}^{2}$ für die Gl. 4 umgerechnet. Die Annahme von $10^{\circ} \mathrm{C}$ wurde getroffen, da diese Temperatur ungefähr der mittleren Grundwassertemperatur in Deutschland entspricht und somit die beste Annäherung an reale In-situ-Bedingungen darstellt (z. B. Menberg et al. 2014; Benz et al. 2015, 2017).

Die effektive hydraulische Durchlässigkeit des Sandsteinblocks kann aus den beiden hydraulischen Größen der Matrix und der Kluft wie folgt berechnet werden (de Marsily 1981; Toublanc et al. 2005):

$k_{\mathrm{eff}}=k_{\mathrm{m}}+k_{\mathrm{fr}}$

Mithilfe dieser Gleichung konnten Zeeb et al. (2010) Insitu hydraulische Kluftöffnungsweiten für einen Sandstein in Saudi-Arabien abschätzen, da die effektive hydraulische Durchlässigkeit dieser Formation durch zahlreiche Pumpversuche und die Matrixpermeabilität an Sandsteinkernen im Labor bestimmt wurde. Das Verhältnis der hydraulischen Durchlässigkeiten zwischen Kluft und Matrix $k_{\mathrm{fr}} / k_{\mathrm{m}}$ beträgt ungefähr $10 \mathrm{zu} 1$ für den untersuchten Wajid-Sandstein (Zeeb et al. 2010) und unterstreicht die Bedeutung der Klüfte für das Strömungsverhalten in diesen Sandsteinen. 


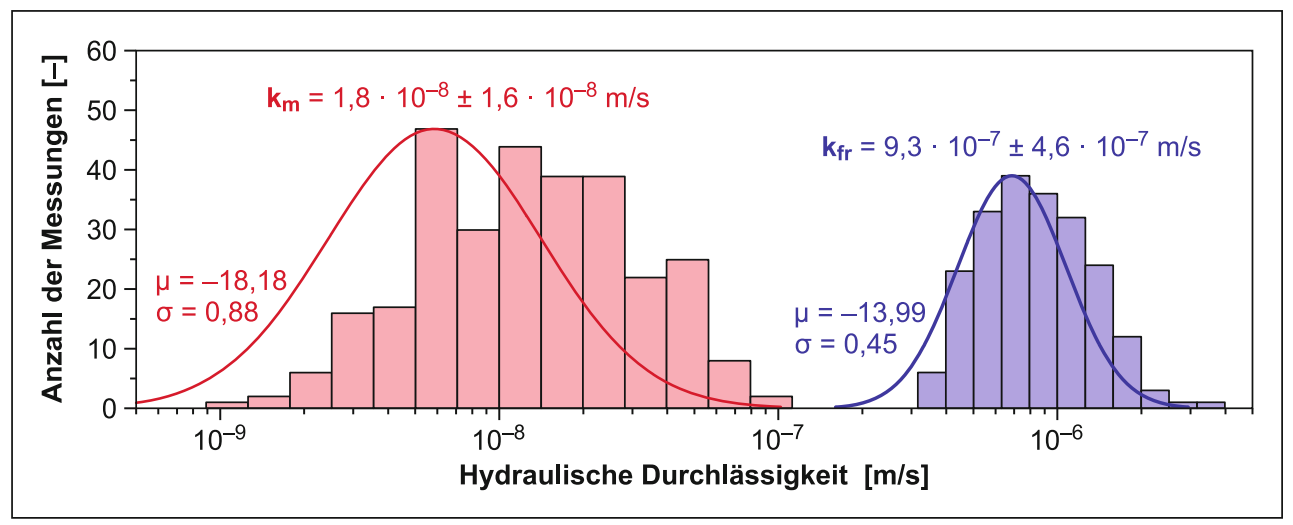

Abb. 4 Häufigkeitsverteilung der bestimmten hydraulischen Durchlässigkeiten für die Sandsteinmatrix (rot) und die Schichtfuge (blau). Für beide Datensätze erfolgte die Anpassung einer Wahrscheinlichkeitsdichtefunktion (rote und blaue Kurve) basierend auf einer logarithmischen Normalverteilung mit den zugehörigen Schätzparametern $\mu$ und $\sigma$

Fig. 4 Frequency distribution of the determined hydraulic conductivities for the sandstone matrix (red) and the bedding joint (blue). A probability density function was fitted to both data sets based on a log-normal distribution with the estimation parameters $\mu$ and $\sigma$

\section{Ergebnisse und Diskussion}

Insgesamt wurden 298 Messpunkte auf der Vorder- und Rückseite des Sandsteinblocks zur Bestimmung der Matrixpermeabilität berücksichtigt. Von ursprünglich 303 Messpunkten (Heidsiek et al. 2020) wurden fünf Messpunkte aus dem Bereich einer sehr kleinskaligen hochpermeablen Struktur auf der Rückseite des Blocks aus dem Datensatz entfernt. Die Ergebnisse der räumlichen Verteilung der gemessenen Matrixpermeabilitäten sind in Abb. 3a für die Vorderseite des Sandsteinblocks dargestellt. Die gemessene durchschnittliche Matrixpermeabilität der äolischen Fazies beträgt 2,5 $\pm 2,2 \mathrm{mD}$, die der fluviatilen Fazies 2,2 $\pm 2,0 \mathrm{mD}$. Mit einem Wertebereich der berechneten hydraulischen Durchlässigkeit $k_{\mathrm{m}}$ von $9,2 \cdot 10^{-10}$ bis $1,0 \cdot 10^{-7} \mathrm{~m} / \mathrm{s}$ sind diese Messwerte vergleichbar mit den Ergebnissen anderer Studien, die für den Flechtinger Sandstein hydraulische Durchlässigkeiten von 1,5 bis $3,0 \cdot 10^{-9} \mathrm{~m} / \mathrm{s}$ (Hassanzadegan et al. 2012) und $3,7 \cdot 10^{-8}$ bis $1,9 \cdot 10^{-7} \mathrm{~m} / \mathrm{s}$ (Cheng und Milsch 2020) angeben. Eine weiterführende Diskussion im Hinblick auf die Diagenese und den Einfluss der Zementation auf die Matrixpermeabilitäten wird von Heidsiek et al. (2020) geführt. In Tab. 1 sind die wichtigsten statistischen Kenngrößen für alle Messwerte zusammengestellt.

Die hydraulische Kluftöffnungsweite wurde an insgesamt 21 Messpunkten entlang der Schichtfuge bestimmt (Abb. 3b). Die mittlere Kluftöffnungsweite beträgt $82 \mu \mathrm{m}$ mit einer Standardabweichung von $12 \mu \mathrm{m}$. Bei den Messungen stand die Kluft unter einer minimalen konstanten Spannung von ungefähr $0,04 \mathrm{MPa}$, die mithilfe von Schraubzwingen aufgebracht wurde (Abb. 1b, Hale et al. 2020), was einer Auflast in einer Tiefe von rund $1,5 \mathrm{~m}$ entspricht. Die gemessenen Werte liegen in einem typischen Bereich für beobachtete Kluftöffnungsweiten in Sandsteinen (z. B. Kel- ler 1998; Baraka-Lokmane und Liedl 2006; Hooker et al. 2009; Cheng et al. 2020).

Mithilfe der Gln. 4 und 5 konnten die jeweiligen hydraulischen Durchlässigkeiten sowohl für die Matrix als auch für die Schichtfuge berechnet werden (Tab. 1). Die Ergebnisse sind ebenfalls in Abb. 4 zusammenfassend dargestellt und zeigen eine deutlich erhöhte hydraulische Durchlässigkeit für die Kluft mit einem Mittelwert von $9,3 \cdot 10^{-7} \mathrm{~m} / \mathrm{s}$ gegenüber einer mittleren hydraulischen Matrixdurchlässigkeit des Sandsteinblocks von nur $1,8 \cdot 10^{-8} \mathrm{~m} / \mathrm{s}$. Daraus ergibt sich ein Verhältnis der hydraulischen Durchlässigkeiten zwischen Kluft und Matrix $k_{\mathrm{fr}} / k_{\mathrm{m}}$ von etwa $52 \mathrm{zu}$ 1 , welches deutlich höher ist als das beobachtete Verhältnis bei Zeeb et al. (2010) für den Wajid-Sandstein von rund 10 zu 1. Neben dem abweichenden tektonischen Setting können diese Unterschiede generell durch die Skalenabhängigkeit hydraulischer Ermittlungsverfahren verursacht werden (s. oben). Auch lässt sich die beobachtete Abweichung damit erklären, dass die Bestimmung der effektiven hydraulischen Durchlässigkeiten bei Zeeb et al. (2010) mithilfe von Pumpversuchen erfolgte, die in einer Tiefe von mehreren hundert Metern durchgeführt wurden und dieses Verhältnis somit kleiner ist als in dieser Studie. Typischerweise wird eine exponentielle Abnahme von Kluftöffnungsweiten mit der Tiefe beobachtet (z. B. Snow 1968; Garritty 1983; Ingebritsen und Manning 1999; Stober und Bucher 2007). Mithilfe von exponentiellen und empirischen Modellen können diese in die Tiefe extrapoliert werden (z.B. Cheema 2015; Achtziger-Zupančič et al. 2017), oder es werden hydromechanische Modellansätze verwendet, die ebenfalls eine exponentielle Abnahme der Kluftöffnungsweite mit zunehmender Spannung nachbilden (z.B. Wang und Cardenas 2016; Kling et al. 2018). Für diese hydromechanischen Modelle werden jedoch wiederum auch mechanische Klufteigenschaften benötigt, wie z.B. der E-Modul $E$, die Ein- 
Tab. 1 Ergebnisse der Messungen der hydraulischen Durchlässigkeit für die Sandsteinmatrix und die Schichtfuge

Table 1 Results of the hydraulic conductivity measurements for the sandstone matrix and the bedding joint

\begin{tabular}{llllll}
\hline & Mittelwert $[\mathrm{m} / \mathrm{s}]$ & Standardabweichung $[\mathrm{m} / \mathrm{s}]$ & Minimum $[\mathrm{m} / \mathrm{s}]$ & Median $[\mathrm{m} / \mathrm{s}]$ & Maximum $[\mathrm{m} / \mathrm{s}]$ \\
\hline Matrix, äolische Fazies $(n=273)$ & $1,8 \cdot 10^{-8}$ & $1,7 \cdot 10^{-8}$ & $9,2 \cdot 10^{-10}$ & $1,4 \cdot 10^{-8}$ & $1,0 \cdot 10^{-7}$ \\
Matrix, fluviatile Fazies $(n=25)$ & $1,7 \cdot 10^{-8}$ & $1,5 \cdot 10^{-8}$ & $2,2 \cdot 10^{-9}$ & $1,3 \cdot 10^{-8}$ & $6,9 \cdot 10^{-8}$ \\
Schichtfuge $(n=210)$ & $9,3 \cdot 10^{-7}$ & $4,6 \cdot 10^{-7}$ & $3,3 \cdot 10^{-7}$ & $8,1 \cdot 10^{-7}$ & $3,2 \cdot 10^{-6}$ \\
\hline
\end{tabular}

dringhärte $H$ und typischerweise auch Informationen über die Kluftrauigkeit oder die Verteilung der mechanischen Kluftöffnungsweiten (z. B. Kling et al. 2018). Castaing et al. (2002) konnten wiederum mit synthetischen hydraulischen Modellen zeigen, dass bei einem Verhältnis der hydraulischen Durchlässigkeit zwischen einem Störungssystem und der Matrix von 100 zu 1 das Störungssystem entscheidend das Fließsystem beeinflusst, während bei einem Verhältnis von $10.000 \mathrm{zu} 1$ das Störungssystem das Fließverhalten im Gestein vollständig kontrolliert. Das hier beobachtete Verhältnis von 52 zu 1 für die Einzelkluft deutet also im Hinblick auf den Reservoirmaßstab auf einen großen Einfluss des Kluftsystems auf das Fließverhalten in diesem Sandstein hin.

Beide Datensätze zeigen eine logarithmische Normalverteilung der hydraulischen Durchlässigkeit sowohl für die Matrix als auch für die Kluft (Abb. 4), die auch bereits für andere Sandsteine beobachtet wurde (z.B. Bloomfield und Williams 1995; Dutton und Willis 1998; Wilson et al. 2015). Die logarithmische Normalverteilung ist eine sehr typische Eigenschaft von Sandsteinen, die sich auch in anderen Gesteinseigenschaften wiederfindet, wie z.B. dem Porenhalsdurchmesser oder der Kluftlänge (z.B. Bloomfield et al. 2001; Zeeb et al. 2013; Wilson et al. 2015).

Vorherige Studien und auch die dargestellten Ergebnisse veranschaulichen deutlich, wie wichtig sowohl Kluftals auch Störungssysteme für das Fließverhalten in Sandsteinen sind und es unzureichend ist, lediglich Matrixpermeabilitäten zur Untersuchung von Reservoiren heranzuziehen. Untersuchungen an Aufschlussanalogien mittels tragbarem Luftpermeameter gekoppelt mit hydromechanischen Modellansätzen, wie beispielsweise dem elasto-plastischen Kontaktmodell von Kling et al. (2018), ermöglichen daher auch eine erste realistische Abschätzung der hydraulischen Eigenschaften mit zunehmender Tiefe, um initiale naturnahe Modellierungen für das zu untersuchende Reservoir durchzuführen. Mithilfe von bereits vorhandenen Daten aus Feldversuchen oder zusätzlichen Durchlässigkeitstests an Probekörpern im Labor können diese Ergebnisse wiederum auch In-situ validiert und u. U. den lokalen Gegebenheiten angepasst werden.

\section{Fazit und Ausblick}

In dieser Studie wurden die hydraulischen Durchlässigkeiten eines Sandsteins mithilfe eines kommerziellen und tragbaren Luftpermeameters sowohl für die Matrix als auch für eine Einzelkluft (Schichtfuge) bestimmt. Die Anwendung des Luftpermeameters erweist sich als praktikabel und einfach, sodass robuste Datensätze für Grundwasserleiter erhoben und statistisch ausgewertet werden können. Der hier untersuchte Sandstein zeigt sowohl für die Matrixpermeabilität als auch für die Kluftöffnungsweite eine typische logarithmische Normalverteilung. Der mit dem Luftpermeameter erhobene Datensatz dient als optimale Grundlage, um mithilfe hydromechanischer Modelle eine erste Abschätzung hydraulischer Eigenschaften mit zunehmender Tiefe zu erreichen. Diese können durch weiterführende In-situUntersuchungen validiert und ggf. an lokale Gegebenheiten angepasst werden, und bilden damit eine Basis für den Aufbau von hydromechanisch gekoppelten Modellen zur Simulation von Reservoiren.

Untersuchungen von Reservoirgesteinsanaloga bilden eine Basis für eine Verbesserung und Optimierung der Simulationsmodelle hinsichtlich ihrer hydraulischen als auch hydromechanischen Eigenschaften, welche ebenfalls mit tragbaren Geräten wie z. B. dem Nadelpenetrometer kombiniert erhoben werden können (Hale et al. 2021). Mithilfe dieser tragbaren Geräte können umfangreiche und robuste Datensätze für Reservoire sowohl im Locker- als auch im Festgestein ermittelt werden. Um eine optimale und weitreichende Verbreitung dieser Daten zu ermöglichen, empfehlen wir den Aufbau von öffentlich zugänglichen Analogdatenbanken für die wichtigsten Reservoirgesteine in Deutschland.

Danksagung Wir bedanken uns für die Vorarbeiten bei Beate Dziuba und Matthias Heidsiek im Rahmen Ihrer Abschlussarbeiten. Des Weiteren möchten wir uns für die wertvollen Anregungen und kritischen Kommentare der beiden anonymen Gutachter bedanken. Diese Studie wurde im Rahmen des GEO:N Verbundvorhabens ResKin (Reaktionskinetik in Reservoirgesteinen: Anwendungsbereite Aufskalierung und Modellierung) durchgeführt und finanziert (Vorhaben-Nr.: 03G0871).

Funding Open Access funding enabled and organized by Projekt DEAL.

Open Access Dieser Artikel wird unter der Creative Commons Namensnennung 4.0 International Lizenz veröffentlicht, welche die Nutzung, Vervielfältigung, Bearbeitung, Verbreitung und Wiedergabe in jeglichem Medium und Format erlaubt, sofern Sie den/die ursprüng- 
lichen Autor(en) und die Quelle ordnungsgemäß nennen, einen Link zur Creative Commons Lizenz beifügen und angeben, ob Änderungen vorgenommen wurden.

Die in diesem Artikel enthaltenen Bilder und sonstiges Drittmaterial unterliegen ebenfalls der genannten Creative Commons Lizenz, sofern sich aus der Abbildungslegende nichts anderes ergibt. Sofern das betreffende Material nicht unter der genannten Creative Commons Lizenz steht und die betreffende Handlung nicht nach gesetzlichen Vorschriften erlaubt ist, ist für die oben aufgeführten Weiterverwendungen des Materials die Einwilligung des jeweiligen Rechteinhabers einzuholen.

Weitere Details zur Lizenz entnehmen Sie bitte der Lizenzinformation auf http://creativecommons.org/licenses/by/4.0/deed.de.

\section{Literatur}

Achtziger-Zupančič, P., Loew, S., Mariéthoz, G.: A new global database to improve predictions of permeability distribution in crystalline rocks at site scale. J. Geophys. Res. Solid Earth 122(5), 3513-3539 (2017)

Altenbockum, M., Berens, K., Enzmann, F., Kersten, M., Rüde, T.R., Wieber, G.H.E.: Fallbeispiel Kluftgrundwasserleiter - Charakterisierung eines Grundwasserleiters im Rotliegendsandstein des Saar-Nahe-Beckens. Grundwasser 23(2), 177-187 (2018)

Bagarello, V., Provenzano, G.: Factors affecting field and laboratory measurement of saturated hydraulic conductivity. Trans. ASAE 39(1), 153-159 (1996)

Bai, M., Elsworth, D.: Modeling of subsidence and stress-dependent hydraulic conductivity for intact and fractured porous media. Rock Mech Rock Engng 27(4), 209-234 (1994)

Baier, A., van Geldern, R., Löhr, G., Subert, H.L., Barth, J.A.C.: Grundwasser in Nürnberg: Wichtige Einheiten und deren Nutzbarkeit. Grundwasser 21(3), 253-266 (2016)

Baraka-Lokmane, S., Liedl, R.: From Micro to Meso: an exercise in determining hydraulic conductivity of fractured sandstone cores from detailed characterization of the fractures. Geophys. J. Int. 166(3), 1440-1453 (2006)

Benz, S.A., Bayer, P., Blum, P.: Global patterns of shallow groundwater temperatures. Environ. Res. Lett. 12(3), 34005 (2017)

Benz, S.A., Bayer, P., Menberg, K., Jung, S., Blum, P.: Spatial resolution of anthropogenic heat fluxes into urban aquifers. Sci. Total. Environ. 524-525, 427-439 (2015)

Bloomfield, J.P., Williams, A.T.: An empirical liquid permeability-gas permeability correlation for use in aquifer properties studies. Q. J. Eng. Geol. Hydrogeol. 28(Supplement 2), S143-S150 (1995)

Bloomfield, J., Gooddy, D., Bright, M., Williams, P.: Pore-throat size distributions in Permo-Triassic sandstones from the United Kingdom and some implications for contaminant hydrogeology. Hydrogeol J 9(3), 219-230 (2001)

Brinkmann, P.J., Leßmann, B., Matzer, S.: Eine „typische“ hydrogeologische Systembeschreibung für den Buntsandstein. Grundwasser 19(3), 181-188 (2014)

Brown, S., Smith, M.: A transient-flow syringe air permeameter. Geophysics 78(5), D307-D313 (2013)

Cao, Y.-B., Feng, X.-T., Yan, E.-C., Chen, G., Lü, F.-F., Ji, H.-B., Song, K.-Y.: Calculation method and distribution characteristics of fracture hydraulic aperture from field experiments in fractured granite area. Rock Mech Rock Eng 49(5), 1629-1647 (2016)

Castaing, C., Genter, A., Bourgine, B., Chilès, J.P., Wendling, J., Siegel, P.: Taking into account the complexity of natural fracture systems in reservoir single-phase flow modelling. J. Hydrol. 266(1-2), 83-98 (2002)

Cheema, T.: Depth dependent hydraulic conductivity in fractured sedimentary rocks-a geomechanical approach. Arab J Geosci 8(8), 6267-6278 (2015)
Cheng, C., Milsch, H.: Permeability variations in Illite-bearing sandstone: effects of temperature and nacl fluid salinity. J. Geophys. Res. Solid Earth 125(9), e2020JB020122 (2020)

Cheng, C., Hale, S., Milsch, H., Blum, P.: Measuring hydraulic fracture apertures: a comparison of methods. Solid Earth 11(6), 2411-2423 (2020)

De Boever, W., Bultreys, T., Derluyn, H., Van Hoorebeke, L., Cnudde, V.: Comparison between traditional laboratory tests, permeability measurements and CT-based fluid flow modelling for cultural heritage applications. Sci. Total. Environ. 554-555, 102-112 (2016)

Dutton, S.P., Willis, B.J.: Comparison of outcrop and subsurface sandstone permeability distribution, lower cretaceous fall river formation, south dakota and wyoming. J. Sediment. Res. 68(5), 890-900 (1998)

Farquharson, J., Heap, M.J., Varley, N.R., Baud, P., Reuschlé, T.: Permeability and porosity relationships of edifice-forming andesites: a combined field and laboratory study. J. Volcanol. Geotherm. Res. 297, 52-68 (2015)

Filomena, C.M., Hornung, J., Stollhofen, H.: Assessing accuracy of gas-driven permeability measurements: a comparative study of diverse Hassler-cell and probe permeameter devices. Solid Earth 5(1), 1-11 (2014)

Fischer, C., Dunkl, I., Von Eynatten, H., Wijbrans, J.R., Gaupp, R.: Products and timing of diagenetic processes in Upper Rotliegend sandstones from Bebertal (North German Basin, Parchim Formation, Flechtingen High, Germany). Geol. Mag. 149(5), 827-840 (2012)

Fischer, C., Gaupp, R., Dimke, M., Sill, O.: A 3D high resolution model of bounding surfaces in aeolian-fluvial deposits: An outcrop analogue study from the Permian Rotliegend, Northern Germany. J. Petroleum Geol. 30(3), 257-273 (2007)

Fleuchaus, P., Schüppler, S., Stemmle, R., Menberg, K., Blum, P.: Aquiferspeicher in Deutschland. Grundwasser - Zeitschrift der Fachsektion Hydrogeologie 26(2), 123-134 (2021)

Garritty, P.: Effect of underground construction on geohydrology of proximate rocks: Report to BRE, Contract No, PECD 7/9/75. University of New Castle, Upon Tyne (1983)

Hale, S., Naab, C., Butscher, C., Blum, P.: Method comparison to determine hydraulic apertures of natural fractures. Rock Mech Rock Eng 53(3), 1467-1476 (2020)

Hale, S., Ries, X., Jaeggi, D., Blum, P.: Mechanical and hydraulic properties of the excavation damaged zone (EDZ) in the Opalinus Clay of the Mont Terri Rock Laboratory, Switzerland. Solid Earth 12, 1581-1600 (2021)

Hanauer, B., Söll, T.: Grundwasserbilanzmodell Benker Sandstein in Mittelfranken/Bayern. Grundwasser 1(3-4), 113-120 (1996)

Hassanzadegan, A., Blöcher, G., Zimmermann, G., Milsch, H.: Thermoporoelastic properties of Flechtinger sandstone. Int. J. Rock Mech. Min. Sci. 49, 94-104 (2012)

Heidsiek, M., Butscher, C., Blum, P., Fischer, C.: Small-scale diagenetic facies heterogeneity controls porosity and permeability pattern in reservoir sandstones. Environ Earth Sci 79(18), 425 (2020)

Hooker, J.N., Gale, J.F.W., Gomez, L.A., Laubach, S.E., Marrett, R., Reed, R.M.: Aperture-size scaling variations in a low-strain opening-mode fracture set, Cozzette Sandstone, Colorado. J Struct Geol 31(7), 707-718 (2009)

Huq, F., Haderlein, S.B., Cirpka, O.A., Nowak, M., Blum, P., Grathwohl, P.: Flow-through experiments on water-rock interactions in a sandstone caused by $\mathrm{CO}_{2}$ injection at pressures and temperatures mimicking reservoir conditions. Appl. Geochem 58, 136-146 (2015)

Huysmans, M., Peeters, L., Moermans, G., Dassargues, A.: Relating small-scale sedimentary structures and permeability in a crossbedded aquifer. J. Hydrol. 361(1-2), 41-51 (2008)

Ingebritsen, S.E., Manning, C.E.: Geological implications of a permeability-depth curve for the continental crust. Geol 27(12), $1107-1110$ (1999) 
Kabuth, A., Dahmke, A., Beyer, C., Bilke, L., Dethlefsen, F., Dietrich, P., Duttmann, R., Ebert, M., Feeser, V., Görke, U.-J., Köber, R., Rabbel, W., Schanz, T., Schäfer, D., Würdemann, H., Bauer, S.: Energy storage in the geological subsurface: dimensioning, risk analysis and spatial planning: the ANGUS+ project. Environ Earth Sci 76(1), 1-17 (2017)

Keller, A.: High resolution, non-destructive measurement and characterization of fracture apertures. Int. J. Rock Mech. Min. Sci. 35(8), 1037-1050 (1998)

Kling, T., Vogler, D., Pastewka, L., Amann, F., Blum, P.: Numerical Simulations and Validation of Contact Mechanics in a Granodiorite Fracture. Rock Mech Rock Eng 51(9), 2805-2824 (2018)

Kühn, M., Tesmer, M., Pilz, P., Meyer, R., Reinicke, K., Förster, A., Kolditz, O., Schäfer, D.: CLEAN: project overview on $\mathrm{CO}_{2}$ largescale enhanced gas recovery in the Altmark natural gas field (Germany). Environ Earth Sci 67(2), 311-321 (2012)

Mallants, D., Mohanty, B.P., Vervoort, A., Feyen, J.: Spatial analysis of saturated hydraulic conductivity in a soil with macropores. Soil Technol. 10(2), 115-131 (1997)

Marczinek, S., Schäfer, W., Peiffer, S.: Identifizierung und Quantifizierung natürlicher Abbauprozesse in einem mit Chlorethenen kontaminierten Grundwasserleiter. Grundwasser 11(3), 207-216 (2006)

de Marsily, G.: Hydrogéologie Quantitative. Masson, Paris (1981)

Menberg, K., Blum, P., Kurylyk, B.L., Bayer, P.: Observed groundwater temperature response to recent climate change. Hydrogeol. Earth Syst. Sci. 18(11), 4453-4466 (2014)

Meurer, M., Banning, A.: Uranmobilisierung im Helgoländer Buntsandstein - Auswirkungen auf die Brack- und Trinkwasserqualität. Grundwasser 24(1), 43-50 (2019)

Montanaro, C., Mayer, K., Isaia, R., Gresse, M., Scheu, B., Yilmaz, T.I., Vandemeulebrouck, J., Ricci, T., Dingwell, D.B.: Hydrothermal activity and subsoil complexity: implication for degassing processes at Solfatara crater, Campi Flegrei caldera. Bull Volcanol 79(83), 1-20 (2017)

New England Research: TinyPerm 3 (2015). https://www.ner.com/ite/ systems/tinyperm3.html, Zugegriffen: 21. Juni 2021

Novakowski, K.S., Lapcevic, P.A.: Field measurement of radial solute transport in fractured rock. Water Resour. Res. 30(1), 37-44 (1994)

Petrie, E.S., Petrie, R.A., Evans, J.P.: Identification of reactivation and increased permeability associated with a fault damage zone using a multidisciplinary approach. J Struct Geol 59, 37-49 (2014)

Rogiers, B., Beerten, K., Smeekens, T., Mallants, D., Gedeon, M., Huysmans, M., Batelaan, O., Dassargues, A.: The usefulness of outcrop-analogue air-permeameter measurements for analysing aquifer heterogeneity: testing outcrop hydrogeological parameters with independent borehole data. Hydrol. Earth Syst. Sci. 17, 5155-5166 (2013)
Rogiers, B., Beerten, K., Smeekens, T., Mallants, D., Gedeon, M., Huysmans, M., Batelaan, O., Dassargues, A.: The usefulness of outcrop analogue air permeameter measurements for analyzing aquifer heterogeneity: quantifying outcrop hydraulic conductivity and its spatial variability. Hydrol. Process. 28(20), 5176-5188 (2014b)

Rogiers, B., Winters, P., Huysmans, M., Beerten, K., Mallants, D., Gedeon, M., Batelaan, O., Dassargues, A.: High-resolution saturated hydraulic conductivity logging of borehole cores using air permeability measurements. Hydrogeol J 22(6), 1345-1358 (2014a)

Sato, H.: Thermodynamic equations of state for fluids. Rev. High Press. Sci. Technol. 15(3), 183-190 (2005)

Schulze-Makuch, D., Carlson, D.A., Cherkauer, D.S., Malik, P.: Scale dependency of hydraulic conductivity in heterogeneous media. Ground Water 37(6), 904-919 (1999)

Snow, D.T.: Rock fracture spacings, openings, and porosities. J. Soil Mech. Found. Div. 94(1), 73-91 (1968)

Stober, I., Bucher, K.: Hydraulic properties of the crystalline basement. Hydrogeol J 15(2), 213-224 (2007)

Toublanc, A., Renaud, S., Sylte, J.E., Clausen, C.K., Eiben, T., Nådland, G.: Ekofisk Field: fracture permeability evaluation and implementation in the flow model. Petroleum Geosci. 11(4), 321-330 (2005)

Wang, L., Cardenas, M.B.: Development of an empirical model relating permeability and specific stiffness for rough fractures from numerical deformation experiments. J. Geophys. Res. Solid Earth 121(7), 4977-4989 (2016)

Weede, M., Hötzl, H.: Strömung und Transport in einer natürlichen Einzelkluft in poröser Matrix-Experimente und Modellierung. Grundwasser 10(3), 137-145 (2005)

Whitaker, F.F., Smart, P.L.: Characterising scale-dependence of hydraulic conductivity in carbonates: evidence from the Bahamas. J. Geochem. Explor. 69-70, 133-137 (2000)

Wilson, T.H., Smith, V., Brown, A.: Developing a model discrete fracture network, drilling, and enhanced oil recovery strategy in an unconventional naturally fractured reservoir using integrated field, image log, and three-dimensional seismic data. AAPG Bull. 99(04), 735-762 (2015)

Zeeb, C., Göckus, D., Bons, P., Al Ajmi, H., Rausch, R., Blum, P.: Fracture flow modelling based on satellite images of the Wajid Sandstone, Saudi Arabia. Hydrogeol. J. 18(7), 1699-1712 (2010)

Zeeb, C., Gomez-Rivas, E., Bons, P.D., Blum, P.: Evaluation of sampling methods for fracture network characterization using outcrops. AAPG Bull. 97(9), 1545-1566 (2013)

Hinweis des Verlags Der Verlag bleibt in Hinblick auf geografische Zuordnungen und Gebietsbezeichnungen in veröffentlichten Karten und Institutsadressen neutral. 